\title{
Existence and uniqueness of periodic orbits in a discrete model on Wolbachia infection frequency
}

https://doi.org/10.1515/anona-2020-0194

Received March 27, 2021; accepted May 24, 2021.

\begin{abstract}
In this paper, we study a discrete model on Wolbachia infection frequency. Assume that a periodic and impulsive release strategy is implemented, where infected males are released during the first $N$ generations with the release ratio $\alpha$, and the release is terminated from $(N+1)$-th generation to $T$-th generation. We find a release ratio threshold denoted by $\alpha^{\star}(N, T)$, and prove the existence of a $T$-periodic solution for the model when $\alpha \in\left(0, \alpha^{*}(N, T)\right)$. For the special case when $N=1$ and $T=2$, we prove that the model has a unique $T$-periodic solution which is unstable when $\alpha \in\left(0, \alpha^{\star}(N, T)\right)$. While $\alpha \geq \alpha^{\star}(N, T)$, no periodic phenomenon occurs and the Wolbachia fixation equilibrium is globally asymptotically stable. Numerical simulations are also provided to illustrate our theoretical results. One main contribution of this work is to offer a new method to determine the exact number of periodic orbits to discrete models.
\end{abstract}

Keywords: Discrete model, Wolbachia infection frequency, Mosquito population, Existence and uniqueness, Periodic orbits

MSC: 92B05, 92D30, 37N25

\section{Introduction}

Aedes mosquitoes harbor maternally inherited bacteria Wolbachia inducing "cytoplasmic incompatibility"(CI), resulting in early developmental arrest of the mosquito embryo when infected males mate with uninfected females [2-4, 24, 29-33]. This qualifies two innovative methods to control mosquito populations, seeding naturally uninfected mosquitoes with infected females repeatedly resulted in Wolbachia invasion of mosquito populations, or releasing infected males only to suppress/eradicate wild mosquito populations through CI. For example, in January 2011, Wolbachia infected adults were released to invade two natural Aedes aegypti populations in Cairns, Australia, reaching a near-fixation in a few months [11,29]. Also, since March 2015, by combining the incompatible and sterile insect techniques (IIT-SIT), Wolbachia-infected male mosquitoes have been released on Shazai Island and Dadaosha Island, south of Guangzhou city. The implementation of IIT-SIT in 2016 and 2017 enabled near-elimination of wild-type Aedes albopictus field populations $[19,47]$. These successful field trials demonstrate the feasibility of area-wide application of Wolbachia release for mosquito vector control.

As a safe and self-sustaining method helping communities around the world prevent the spread of mosquito-borne disease, various mathematical models have been developed to study the dynamics of mosquito populations with Wolbachia interference, we refer to [10, 12-14, 21-23, 25, 35, 39-43] for Wolbachia invasion models by simultaneously releasing both infected females and males, and to [5, 15-18, 20, 34, 36-

Bo Zheng, Center for Applied Mathematics, College of Mathematics and Information Sciences, Guangzhou University, Guangzhou, 510006, China, E-mail: zhengbo611@outlook.com

*Corresponding Author: Jianshe Yu, Center for Applied Mathematics, College of Mathematics and Information Sciences, Guangzhou University, Guangzhou, 510006, China, E-mail: jsyu@gzhu.edu.cn 
38, 44-46] for population suppression models by only releasing infected/sterile male mosquitoes. Very recently, by employing the modeling idea firstly introduced in [34] which included the released male mosquitoes as a control function, the interactive dynamical behavior of wild and sterile mosquitoes where the releases of sterile males are impulsive and periodic were studied in [37, 38, 44]. Assume that the time proceeds in continuous steps, the models in $[37,38,44]$ count the abundance of wild mosquitoes in two ordinary differential equations continually switching between each other. By solving explicitly the two ordinary differential equations and obtaining the derivative of the corresponding Poincare map, the exact number of periodic solutions was counted in [37, 38, 44], together with the stability analysis. All current theoretical results imply that monitoring of mosquito population dynamics in cages or semi-field labs prior to Wolbachia releases is necessary and helpful for designing effective release strategies.

Cage experiments monitor the population dynamics with non-overlapping generations, i.e., mosquitoes can be counted generation by generation. For such populations, we can track frequency rather than number, where the discrete model is the first choice due to its easy tractability mathematically. The first discrete model for Wolbachia infection frequency in mosquitoes can be traced back to 1959, when Caspari and Watson were inspired by the evolutionary importance of CI in mosquitoes [6]. The second discrete model, to our best knowledge, can be credited to Fine in 1978 [8]. Hoffmann and Turelli have carried out systematic studies on Wolbachia spreading in Drosophila simulans since 1990's [7, 26-28]. All these models produce "bistable" dynamics, with the existence of an unstable equilibrium, below which the infections tend to be eliminated, and above which Wolbachia invasion is guaranteed to be successful.

Unlike the discrete models proposed in the above literatures, in this paper, we develop a new discrete model for Wolbachia release in cage mosquito populations when extra infected males are released at every generation to promote Wolbachia invasion. This has been proved to be effective in lab experiments [3] that continuously inundative releases of males at every generation can promote Wolbachia fixation by suppressing the effective matings between uninfected females and uninfected males. When the time proceeds in discrete steps, as in [37, 38, 44], we also assume that a periodic and impulsive release strategy is implemented, where infected males are released during the first $N$ generations with the release ratio $\alpha$, and the releases are terminated from the $(N+1)$-th generation to the $T$-th generation with $T \geq N+1$. Hence, the model constantly switches between two difference equations.

Along the lines in $[37,38,44]$, we find a release ratio threshold, $\alpha^{\star}(N, T)$, and prove the existence of a periodic solution to the model when $\alpha \in\left(0, \alpha^{\star}(N, T)\right)$. However, the methods used in $[37,38,44]$ to prove the uniqueness of the periodic solution are not applicable anymore due to the absence of the explicit expressions of the solution. For the special case $N=1$ and $T=2$ which means that Wolbachia infected male mosquitoes are released every other generation, we prove the uniqueness of the periodic solution. By calculating the derivative of the Poincaré map at possible fixed points as what have been done in [37, 38, 44], we prove the uniqueness of fixed points by the qualitative analysis of a quartic polynomial, as well as their stability proofs. However, the uniqueness of periodic solutions remains open when the release function is a general $T$-periodic function with $T>2$. The purpose of this paper is to develop innovative methods for counting the exact number of periodic solutions to discrete models.

\section{A discrete model and main results}

For model development, based on experimental observations in [2, 32], we assume (i) perfect maternal transmission, all offspring from infected females are infected, (ii) complete CI, eggs produced by the incompatible cross do not hatch, and (iii) equal sex determination, the male-to-female birth ratio is always 1:1, no matter whether their mothers are infected or uninfected $[1,9]$.

Assume that there are $I_{n}$ infected mosquitoes and $U_{n}$ uninfected mosquitoes in the $n$-th generation. The infection frequency is denoted by $x_{n}=I_{n} /\left(I_{n}+U_{n}\right)$. The production of mosquitoes in the next generation is interfered by the extra release of infected males at the amount of $m_{n}\left(I_{n}+U_{n}\right)$. The release of these infected males reduces the production rate and also modifies the infection frequency, but not the sex ratio, in the next 
generation. Since the infection status of infected offspring does not depend on paternal infection status, the proportion of infected mosquitoes at the $(n+1)$-th generation is $\left(1-s_{f}\right) x_{n}$, where $s_{f}$ is the relative fitness cost of infected females which means that if the fitness of Wolbachia-free mosquitoes is 1 , then the fitness of infected mosquitoes is $1-s_{f}$. Here, $s_{f}$ includes the fitness cost induced from Wolbachia infection such as reduction in fecundity, egg viability, and mean longevity [3, 11, 24, 29, 32].

Regarding the uninfected offspring, we must account for CI. With the assumption of complete CI and random mating $[6,8,26,28]$, the hatch rate for uninfected embryos is 1 if the father is uninfected with mating probability being $\frac{1-x_{n}}{1+m_{n}}$, but 0 if the father is infected with mating probability equaling to $\frac{x_{n}+m_{n}}{1+m_{n}}$. Hence, the proportion of uninfected mosquitoes at the $(n+1)$-th generation is $\frac{1-x_{n}}{1+m_{n}} \cdot\left(1-x_{n}\right)$. This leads to the relation of the infection frequency between two successive generations as

$$
x_{n+1}=\frac{\left(1-s_{f}\right)\left(1+m_{n}\right) x_{n}}{x_{n}^{2}-\left[1+s_{f}-m_{n}\left(1-s_{f}\right)\right] x_{n}+1} .
$$

Despite its simple form, model (2.1) can generate complex dynamical behavior to illuminate general release strategies with the release ratios being different at every generation. When $m_{n} \equiv \alpha$, model (2.1) offers

$$
\Delta x_{n}=x_{n+1}-x_{n}=\frac{x_{n}\left(1-x_{n}\right)\left(x_{n}+\alpha\left(1-s_{f}\right)-s_{f}\right)}{x_{n}^{2}-\left[1+s_{f}-\alpha\left(1-s_{f}\right)\right] x_{n}+1}, \quad n=1,2, \cdots .
$$

Define

$$
\alpha_{1}^{\star}=\frac{s_{f}}{1-s_{f}} .
$$

We can easily obtain the following conclusions by using the argument in [25, 35].

Theorem 2.1. For $\alpha \geq 0$, model (2.2) admits a trivial equilibrium point $x_{0}^{\star}=0$ and a Wolbachia fixation equilibrium point $x_{1}^{\star}=1$. Furthermore,

(1) if $\alpha \in\left[0, \alpha_{1}^{\star}\right)$, then model (2.2) admits an unstable equilibrium point $\hat{x}(\alpha)=s_{f}-\alpha\left(1-s_{f}\right)$, which defines a threshold value on the initial frequency $x_{1}$ : if $x_{1} \in(0, \hat{x}(\alpha))$, then $x_{n} \rightarrow 0$ as $n \rightarrow \infty$, whereas for $x_{1} \in$ $(\hat{x}(\alpha), 1), x_{n} \rightarrow 1$ as $n \rightarrow \infty$.

(2) if $\alpha \geq \alpha_{1}^{\star}$, then $\Delta x_{n}>0$ for any $x_{1} \in(0,1)$, i.e., the Wolbachia fixation equilibrium point $x_{1}^{\star}=1$ is globally asymptotically stable.

Case $\alpha \equiv 0$ corresponds to the model introduced in $[6,8,26,28]$, which admits an unstable equilibrium point $s_{f}$, and model (2.1) also shows a bistable dynamics: when $x_{1} \in\left(0, s_{f}\right),\left\{x_{n}\right\}$ strictly decreases to 0 as $n \rightarrow \infty$, and when $x_{1} \in\left(s_{f}, 1\right),\left\{x_{n}\right\}$ strictly increases to 1 . However, this clean and clear bistable dynamics disappears when $m_{n}$ is not a constant function. To see this, we assume that an impulsive and periodic release strategy is implemented: in each release period $T$, Wolbachia-infected male mosquitoes are released during the first $N$ generations with $m_{n}=\alpha$ for $n \in Z(1+k T, k T+N)$, and the releases are terminated from $(N+1)$-th to $T$-th generation with $m_{n}=0$ for $n \in Z(k T+N+1,(k+1) T)$. Then, the infection frequency follows

$$
x_{n+1}=\frac{\left(1-s_{f}\right)(1+\alpha) x_{n}}{x_{n}^{2}-\left[1+s_{f}-\alpha\left(1-s_{f}\right)\right] x_{n}+1}=F\left(x_{n}, \alpha\right)
$$

for $n \in Z(1+k T, k T+N)$, and

$$
x_{n+1}=\frac{\left(1-s_{f}\right) x_{n}}{x_{n}^{2}-\left(1+s_{f}\right) x_{n}+1}=G\left(x_{n}, \alpha\right)
$$

for $n \in Z(k T+N+1,(k+1) T)$.

Let $\left\{x_{n}\right\}_{n=1}^{\infty}=\left\{x_{n}(1, u)\right\}_{n=1}^{\infty}$ denote the solution of system (2.3)-(2.4) with initial value $x_{1}=u>0$. Then $\left\{x_{n}\right\}_{n=2}^{N+1}$ satisfies (2.3) with the initial value $x_{1}=u$ and $x_{2}=F(u, \alpha)$, which yields

$$
\bar{h}(u, \alpha)=x_{N+1}=F^{(N)}(u, \alpha)
$$


by induction. Initiated from $x_{N+1},\left\{x_{n}\right\}_{n=N+2}^{T+1}$ satisfies (2.4) with $x_{n}=G^{(n-N-1)}\left(x_{N+1}\right)$. Hence,

$$
h(u, \alpha)=x_{T+1}=G^{(T-N)}\left(x_{N+1}\right)=G^{(T-N)}\left(F^{(N)}(u, \alpha)\right) .
$$

Thus, the dynamical behavior of solutions to system (2.3)-(2.4) depends on the continually switching between equations (2.3) and (2.4). It is obvious that $h(0, \alpha)=\bar{h}(0, \alpha)=0$ and $h(1, \alpha)=\bar{h}(1, \alpha)=1$, which implies that 0 and 1 are two fixed points of $h$. In order to find the nontrivial $T$-periodic solution of system (2.3)-(2.4), we only need to find the initial value $u>0$ such that $h(u, \alpha)=u$. We introduce

$$
\alpha^{\star}(N, T)=\frac{1}{\left(1-s_{f}\right)^{T / N}}-1,
$$

and obtain the following result for the existence of $T$-periodic solution of system (2.3)-(2.4), which is one of our main results in this paper.

Theorem 2.2. When $\alpha \in\left(0, \alpha^{\star}(N, T)\right)$, system (2.3)-(2.4) has at least one T-periodic solution.

Example 2.1. We assume that Wolbachia infection causes a mild fitness cost to the host mosquito with $s_{f}=0.3$, and $N=3, T=7$, which yield $\alpha^{\star}(N, T) \approx 1.2985$ and system (2.3)-(2.4) is a discrete T-periodic system

$$
x_{n+1}=\left\{\begin{array}{cc}
\frac{0.7(1+\alpha) x_{n}}{x_{n}^{2}-(1.3-0.7 \alpha) x_{n}+1}, & n \in Z(7 k+1,7 k+3), \\
\frac{0.7 x_{n}}{x_{n}^{2}-1.3 x_{n}+1}, & n \in Z(7 k+4,7(k+1)),
\end{array}\right.
$$

for $k=0,1,2, \cdots$. Figure 1 plots $h(u, \alpha)-u$ against $u$ by randomly selecting 400 samples for $u$ in $[0,1]$, and then solving equation (2.7) in Matlab with the initial value $u$. Panel (A) not only shows the existence of the periodic solution, but also manifests that the periodic solution is unique when $\alpha<\alpha^{*}(N, T)$. Panel $(B)$, and our numerical trials not shown here, shows that the value $\alpha^{\star}(N, T)$ defines a threshold value on the release ratio: when the release ratio $\alpha \geq \alpha^{\star}(N, T)$, one has $h(u, \alpha)>u$, and hence equation (2.7) has no periodic solution.

Although Example 2.1 proves numerically the uniqueness of the periodic solution when $\alpha<\alpha^{\star}(N, T)$, it
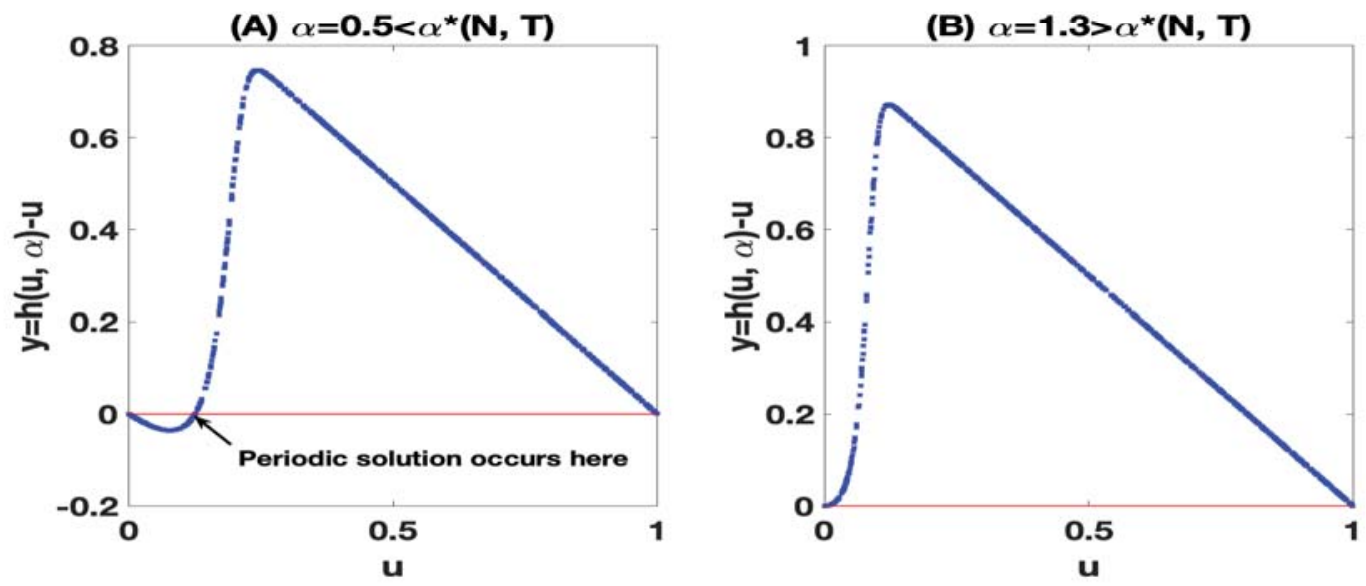

Fig. 1: Numerical implications on the threshold quality of $\alpha^{\star}(N, T)$ regarding the existence and uniqueness of the periodic solution to system (2.3)-(2.4). (A) When $\alpha<\alpha^{\star}(N, T)$, system (2.3)-(2.4) has a unique periodic solution with the initial value $u$ lying in $(0,1)$. (B) When $\alpha \geq \alpha^{\star}(N, T)$, no periodic phenomenon occurs for all $u \in(0,1)$.

seems that the methods in $[37,38,44]$ for proving the uniqueness of fixed points of $h$ are not applicable since neither $\bar{h}(u, \alpha)$ nor $h(u, \alpha)=h(\bar{h}(u, \alpha))$ to system (2.3)-(2.4) can be explicitly expressed in $u$ and $\bar{h}(u, \alpha)$, respectively. Instead, the two functions, defined in (2.5) and (2.6), are composite functions of $F$ and $G$. Their 
explicit expressions require the calculation of $F^{(N)}(u, \alpha)$ and $G^{(T-N)}\left(F^{(N)}(u, \alpha)\right)$, which is a mission impossible for general $N$ and $T$. We take the simplest case out in this paper as our first try, when male mosquitoes are periodically and impulsively released every other generation with $N=1$ and $T=2$, i.e.,

$$
m_{n}=\left\{\begin{array}{cc}
\alpha, & n=2 k+1, \\
0, & n=2(k+1),
\end{array}\right.
$$

for $k=0,1,2, \cdots$. Thus, model (2.1) consists of

$$
x_{n+1}=\frac{\left(1-s_{f}\right)(1+\alpha) x_{n}}{x_{n}^{2}-\left[1+s_{f}-\alpha\left(1-s_{f}\right)\right] x_{n}+1}, \quad n=2 k+1,
$$

and

$$
x_{n+1}=\frac{\left(1-s_{f}\right) x_{n}}{x_{n}^{2}-\left(1+s_{f}\right) x_{n}+1}, \quad n=2(k+1),
$$

and $\bar{h}(u, \alpha)=F(u, \alpha)$ and $h(u, \alpha)=G(\bar{h}(u, \alpha))=G(F(u, \alpha))$. By reducing the $\alpha^{\star}(N, T)$ to the release ratio threshold

$$
\alpha^{\star}=\alpha^{\star}(1,2)=\frac{1}{\left(1-s_{f}\right)^{2}}-1,
$$

we have the following results for a complete dynamical description of system (2.8)-(2.9).

Theorem 2.3. When $\alpha \in\left(0, \alpha^{\star}\right)$, system (2.8)-(2.9) has a unique periodic solution initiated from $u^{\star} \in\left(0, s_{f}\right)$, which is unstable. Furthermore, both the trivial equilibrium point $x_{0}^{\star}=0$ and the Wolbachia fixation equilibrium point $x_{1}^{*}=1$ are locally asymptotically stable.

Theorem 2.4. When $\alpha \geq \alpha^{\star}$, system (2.8)-(2.9) has no periodic solution, and equilibrium $x_{1}^{\star}$ is globally asymptotically stable.

We prove Theorems 2.2, 2.3 and 2.4 in the following section.

\section{Proofs of the main results}

\subsection{Proof of Theorem 2.2}

The existence of periodic solutions to system (2.3)-(2.4) depends on whether the function $h(u, \alpha)-u$ is signchanging or not. It is obvious that $h(0, \alpha)=\bar{h}(0, \alpha)=0$ and $h(1, \alpha)=\bar{h}(1, \alpha)=1$ for any $\alpha>0$. Comparisons among $h(u, \alpha), \bar{h}(u, \alpha)$ and $u$ directly deduced from Theorem 2.1 when $\alpha \in\left(0, \alpha_{1}^{\star}\right)$ are listed below:

$$
\begin{aligned}
& \bar{h}(u, \alpha)<u, h(u, \alpha)<\bar{h}(u, \alpha) \Rightarrow h(u, \alpha)<u \quad \text { for } u \in(0, \hat{x}(\alpha)) . \\
& \bar{h}(\hat{x}(\alpha), \alpha)=\hat{x}(\alpha), h(\hat{x}(\alpha), \alpha)<\hat{x}(\alpha) . \\
& \bar{h}(u, \alpha)>u, h(u, \alpha)<\bar{h}(u, \alpha) \quad \text { for } u \in\left(\hat{x}(\alpha), s_{f}\right) . \\
& \bar{h}\left(s_{f}, \alpha\right)>s_{f} \Rightarrow h\left(s_{f}, \alpha\right)>s_{f} . \\
& \bar{h}(u, \alpha)>u, h(u, \alpha)>\bar{h}(u, \alpha) \Rightarrow h(u, \alpha)>u \quad \text { for } u \in\left(s_{f}, 1\right) .
\end{aligned}
$$

Similarly, when $\alpha \geq \alpha_{1}^{\star}$, we have

$$
\begin{aligned}
& \bar{h}(u, \alpha)>u, h(u, \alpha)<\bar{h}(u, \alpha) \text { for } u \in\left(0, s_{f}\right) . \\
& \bar{h}\left(s_{f}, \alpha\right)>s_{f} \Rightarrow h\left(s_{f}, \alpha\right)>s_{f} . \\
& \bar{h}(u, \alpha)>u, h(u, \alpha)>\bar{h}(u, \alpha) \Rightarrow h(u, \alpha)>u \quad \text { for } u \in\left(s_{f}, 1\right) .
\end{aligned}
$$

The relations (3.1) and (3.2) imply that when $u \in\left[s_{f}, 1\right)$, it holds that $h(u, \alpha)>u$ for any $\alpha>0$, and so system (2.3)-(2.4) does not possess periodic solutions. Also, when $\alpha \in\left(0, \alpha_{1}^{*}\right),(3.1)$ shows that $h(u, \alpha)<u$ for 
$u \in(0, \hat{x}(\alpha))$, and $h(u, \alpha)>u$ for $u \in\left[s_{f}, 1\right)$. Therefore, when $\alpha \in\left(0, \alpha_{1}^{\star}\right)$, system (2.3)-(2.4) has at least one periodic solution with $u$ lying in $\left(\hat{x}(\alpha), s_{f}\right)$.

For the case $\alpha \geq \alpha_{1}^{\star}$, the sign of $h(u, \alpha)-u$ for $u \in\left(0, s_{f}\right)$ is uncertain, which leads us to consider $h_{u}^{\prime}(0, \alpha)$. Noticing that $h(0, \alpha)=\bar{h}(0, \alpha)=0$, from (2.3) and (2.5), we have

$$
\bar{h}_{u}^{\prime}(0, \alpha)=\lim _{u \rightarrow 0} \frac{\bar{h}(u, \alpha)}{u}=\lim _{u \rightarrow 0} \frac{F^{(N)}(u, \alpha)}{F^{(N-1)}(u, \alpha)} \cdot \frac{F^{(N-1)}(u, \alpha)}{F^{(N-2)}(u, \alpha)} \cdots \frac{F(u, \alpha)}{u},
$$

where we use $h_{u}^{\prime}(u, \alpha)$ to denote the partial derivative of $\bar{h}(u, \alpha)$ with respect to $u$.

Noticing that $F(0, \alpha)=F^{(2)}(0, \alpha)=\cdots=F^{(N)}(0, \alpha)=0$, by denoting $F(u, \alpha)=F^{(1)}(u, \alpha)$ and $u=F^{(0)}(u, \alpha)$, we have

$$
\begin{aligned}
\lim _{u \rightarrow 0} \frac{F^{(i)}(u, \alpha)}{F^{(i-1)}(u, \alpha)} & =\lim _{u \rightarrow 0} \frac{F\left(F^{(i-1)}(u, \alpha)\right)}{F^{(i-1)}(u, \alpha)} \\
& =\lim _{u \rightarrow 0} \frac{\left(1-s_{f}\right)(1+\alpha)}{\left[F^{(i-1)}(u, \alpha)\right]^{2}-\left[1+s_{f}-\alpha\left(1-s_{f}\right)\right] F^{(i-1)}(u, \alpha)+1} \\
& =\left(1-s_{f}\right)(1+\alpha),
\end{aligned}
$$

for $i=1,2, \cdots, N$. Thus (3.3) leads to

$$
\bar{h}_{u}^{\prime}(0, \alpha)=\left(1-s_{f}\right)^{N}(1+\alpha)^{N} .
$$

Similarly, from (2.4) and (2.6), we have

$$
\lim _{u \rightarrow 0} \frac{h(u, \alpha)}{\bar{h}(u, \alpha)}=\lim _{u \rightarrow 0} \frac{G^{(T-N)}(\bar{h}(u, \alpha))}{G^{(T-N-1)}(\bar{h}(u, \alpha))} \cdot \frac{G^{(T-N-1)}(\bar{h}(u, \alpha))}{G^{(T-N-2)}(\bar{h}(u, \alpha))} \cdots \frac{G(\bar{h}(u, \alpha))}{\bar{h}(u, \alpha)}=\left(1-s_{f}\right)^{T-N} .
$$

Combining (3.4) and (3.5), we reach

$$
h_{u}^{\prime}(0, \alpha)=\lim _{u \rightarrow 0} \frac{h(u, \alpha)}{u}=\lim _{u \rightarrow 0} \frac{h(u, \alpha)}{\bar{h}(u, \alpha)} \cdot \frac{\bar{h}(u, \alpha)}{u}=\left(1-s_{f}\right)^{T}(1+\alpha)^{N} .
$$

Then, we have $h_{u}^{\prime}(0, \alpha)<1$ if and only if $\alpha<\alpha^{\star}(N, T)$. Hence, when $\alpha<\alpha^{\star}(N, T)$, there is sufficiently small $\delta>0$ such that $h(u, \alpha)<u$ for $u \in(0, \delta)$. Together with $h\left(s_{f}, \alpha\right)>s_{f}$, there must exist $u^{\star} \in\left(\delta, s_{f}\right)$ such that

$$
h\left(u^{*}, \alpha\right)=u^{\star}, h_{u}^{\prime}\left(u^{*}, \alpha\right) \geq 1 \text { and } h(u, \alpha)<u \text { for } u \in\left(0, u^{\star}\right) .
$$

Therefore, When $\alpha \in\left[\alpha_{1}^{\star}, \alpha^{\star}(N, T)\right)$, system (2.3)-(2.4) has at least one periodic solution with $u$ lying in $\left(0, s_{f}\right)$. Theorem 2.2 has been proved.

\subsection{Proof of Theorem 2.3}

Let $\left\{x_{n}\right\}_{n=1}^{\infty}=\left\{x_{n}\left(1, x_{1}\right)\right\}_{n=1}^{\infty}$ be the solution of system (2.8)-(2.9) initiated from $x_{1}=u \in(0,1)$. Then

$$
\bar{h}(u, \alpha)=x_{2}=\frac{\left(1-s_{f}\right)(1+\alpha) u}{u^{2}-\left[1+s_{f}-\alpha\left(1-s_{f}\right)\right] u+1}=\frac{P(u, \alpha)}{Q(u, \alpha)},
$$

and

$$
h(u, \alpha)=x_{3}=\frac{\left(1-s_{f}\right) \bar{h}(u, \alpha)}{\bar{h}^{2}(u, \alpha)-\left(1+s_{f}\right) \bar{h}(u, \alpha)+1}=\frac{R(\bar{h}(u, \alpha))}{S(\bar{h}(u, \alpha))} .
$$

We begin with calculating $h_{u}^{\prime}(u, \alpha)$. From (3.7), we have

$$
\bar{h}_{u}^{\prime}(u, \alpha)=\frac{P_{u}^{\prime}(u, \alpha)}{Q_{u}(u, \alpha)}-\frac{P(u, \alpha)}{Q^{2}(u, \alpha)} Q_{u}^{\prime}(u, \alpha) \stackrel{u \neq 0}{=} \frac{\left[P_{u}^{\prime}(u, \alpha)-Q_{u}^{\prime}(u, \alpha) \bar{h}(u, \alpha)\right] \cdot \bar{h}(u, \alpha)}{P(u, \alpha)} .
$$


Similarly, from (3.8), we get

$$
\begin{aligned}
h_{u}^{\prime}(\bar{h}(u, \alpha))= & \frac{R^{\prime}(\bar{h}(u, \alpha))}{S(\bar{h}(u, \alpha))}-\frac{R(\bar{h}(u, \alpha))}{S^{2}(\bar{h}(u, \alpha))} S^{\prime}(\bar{h}(u, \alpha)) \\
\underline{\underline{\bar{h}(u) \neq 0}} & \frac{\left[R^{\prime}(\bar{h}(u, \alpha))-S^{\prime}(\bar{h}(u, \alpha)) h(u, \alpha)\right] \cdot h(u, \alpha)}{R(\bar{h}(u, \alpha))} .
\end{aligned}
$$

Combining (3.9) and (3.10), for $u \in \Gamma=\left\{u \in\left(0, s_{f}\right): h(u, \alpha)=u\right\}$, we reach

$$
\begin{aligned}
h_{u}^{\prime}(u, \alpha) & =\frac{\left[R^{\prime}(\bar{h}(u, \alpha))-S^{\prime}(\bar{h}(u, \alpha)) u\right] u}{R(\bar{h}(u, \alpha))} \cdot \frac{\left[P^{\prime}(u, \alpha)-Q^{\prime}(u, \alpha) \bar{h}(u, \alpha)\right] \bar{h}(u, \alpha)}{P(u, \alpha)} \\
& =\frac{\left\{1-s_{f}-\left[2 \bar{h}(u, \alpha)-\left(s_{f}+1\right)\right] u\right\} \cdot\left\{\left(1-s_{f}\right)(1+\alpha)-\left[2 u-\left(s_{f}+1-\alpha\left(1-s_{f}\right)\right)\right] \bar{h}(u, \alpha)\right\}}{\left(1-s_{f}\right)^{2}(1+\alpha)}
\end{aligned}
$$

where

$$
\begin{aligned}
1-s_{f}-\left[2 \bar{h}(u, \alpha)-\left(s_{f}+1\right)\right] u & =\frac{\left(1-s_{f}\right) Q(u, \alpha)-2 u P(u, \alpha)+\left(s_{f}+1\right) u Q(u, \alpha)}{Q(u, \alpha)} \\
& =\frac{(1-u) \cdot\left\{-\left(s_{f}+1\right) u^{2}+\left[\alpha\left(1-s_{f}\right)^{2}+1+s_{f}^{2}\right] u+1-s_{f}\right\}}{Q(u, \alpha)},
\end{aligned}
$$

and

$$
\left(1-s_{f}\right)(1+\alpha)-\left[2 u-\left(s_{f}+1-\alpha\left(1-s_{f}\right)\right)\right] \bar{h}(u, \alpha)=\frac{(1-u)(1+u)\left(1-s_{f}\right)(1+\alpha)}{Q(u, \alpha)} .
$$

Therefore, for $u \in \Gamma$, we calculate $h_{u}^{\prime}(u, \alpha)$ as

$$
h_{u}^{\prime}(u, \alpha)=\frac{(1-u)^{2}(1+u)\left\{-\left(s_{f}+1\right) u^{2}+\left[\alpha\left(1-s_{f}\right)^{2}+1+s_{f}^{2}\right] u+1-s_{f}\right\}}{\left(1-s_{f}\right)\left[u^{2}-\left(s_{f}+1-\alpha\left(1-s_{f}\right)\right) u+1\right]^{2}} .
$$

Further computation from (3.11) offers

$$
h_{u}^{\prime}(u, \alpha)<1 \Leftrightarrow u g(u, \alpha)<0 \Leftrightarrow g(u, \alpha)<0,
$$

for $u \in \Gamma$, where

$$
g(u, \alpha)=a_{0}+a_{1} u+a_{2} u^{2}+a_{3} u^{3}+a_{4} u^{4},
$$

with

$$
\begin{gathered}
a_{0}=-\left(1-s_{f}\right)^{2} \alpha+2+s_{f}-s_{f}^{2}, \quad a_{1}=-\left(1-s_{f}\right)^{3} \alpha^{2}+\left(2 s_{f}^{3}-3 s_{f}^{2}+1\right) \alpha+s_{f}^{3}+s_{f}-6, \\
a_{2}=3\left(1-s_{f}\right)\left(1+s_{f}-\alpha\left(1-s_{f}\right)\right), \quad a_{3}=\alpha\left(1-s_{f}\right)^{2}+\left(1+s_{f}\right)^{2}, \quad a_{4}=-\left(1+s_{f}\right)<0 .
\end{gathered}
$$

The following three lemmas are devoted to qualitative properties of the quartic polynomial $g(u, \alpha)$.

Lemma 3.1. The function $h_{u}^{\prime}(u, \alpha)$, or equivalently $g(u, \alpha)$, is strictly decreasing with respect to $\alpha$ for all $\alpha \geq 0$ and $u \in \Gamma$.

Proof. Rewrite (3.11) as

$$
h_{u}^{\prime}(u, \alpha)=\frac{(1-u)^{2}(1+u) f(u, \alpha)}{\left(1-s_{f}\right) Q^{2}(u, \alpha)}:=\frac{(1-u)^{2}(1+u)}{\left(1-s_{f}\right)} K(u, \alpha)
$$

Since

$$
\begin{aligned}
\frac{\partial K(u, \alpha)}{\partial \alpha} & =\frac{u\left(1-s_{f}\right)\left[\left(1-s_{f}\right) Q(u, \alpha)-2 f(u, \alpha)\right]}{Q^{4}(u, \alpha)} \\
& =\frac{u\left(1-s_{f}\right)\left[\left(s_{f}+3\right) u^{2}-\left(\left(1-s_{f}\right)^{2} \alpha+3+s_{f}^{2}\right) u+s_{f}-1\right]}{Q^{4}(u, \alpha)}
\end{aligned}
$$




$$
\begin{aligned}
& \leq \frac{u\left(1-s_{f}\right)\left[\left(s_{f}+3\right) u^{2}-\left(3+s_{f}^{2}\right) u+s_{f}-1\right]}{Q^{4}(u, \alpha)} \\
& =\frac{u\left(1-s_{f}\right)\left[s_{f} u\left(u-s_{f}\right)+3 u(u-1)+s_{f}-1\right]}{Q^{4}(u, \alpha)}<0
\end{aligned}
$$

for $u \in\left(0, s_{f}\right)$, the proof is complete.

Lemma 3.2. For all $\alpha \geq 0$, the function $g(u, \alpha)$ is strictly decreasing with respect to $u$ for all $u \in(0,1)$.

Proof. From $g_{u}^{\prime}(u, \alpha)=a_{1}+2 a_{2} u+3 a_{3} u^{2}+4 a_{4} u^{3}$, we have $g_{u}^{\prime}(1, \alpha)=-(1+\alpha)^{2}\left(1-s_{f}\right)^{3}<0$ and we claim

$$
g_{u}^{\prime}(0, \alpha)=a_{1}<0 .
$$

In fact, by viewing $a_{1}$ as a function of $\alpha$, we have

$$
a_{1}=a_{1}(\alpha)=-\left(1-s_{f}\right)^{3} \alpha^{2}+\left(2 s_{f}^{3}-3 s_{f}^{2}+1\right) \alpha+s_{f}^{3}+s_{f}-6 .
$$

The discriminant of the equation $a_{1}(\alpha)=0$ w.r.t. $\alpha$ is

$$
\left(2 s_{f}^{3}-3 s_{f}^{2}+1\right)^{2}+4\left(1-s_{f}\right)^{3}\left(s_{f}^{3}+s_{f}-6\right)=\left(1-s_{f}\right)^{3}\left(7 s_{f}-23\right)<0,
$$

together with $a_{1}(0)=s_{f}^{3}+s_{f}-6<0$, and hence (3.14) holds.

We next prove $g_{u}^{\prime}(u, \alpha)<0$ for all $u \in(0,1)$ by exploring the qualitative behavior of the function $g_{u}^{\prime \prime}(u, \alpha)$. From

$$
\begin{aligned}
\frac{g_{u}^{\prime \prime}(u, \alpha)}{6} & =\frac{a_{2}}{3}+a_{3} u+2 a_{4} u^{2} \\
& =-2\left(1+s_{f}\right) u^{2}+\left[\alpha\left(1-s_{f}\right)^{2}+\left(1+s_{f}\right)^{2}\right] u+\left(1-s_{f}\right)\left[1+s_{f}-\alpha\left(1-s_{f}\right)\right],
\end{aligned}
$$

we have $g_{u}^{\prime \prime}(1, \alpha)=0$ and the $x$-coordinate of the vertex of $g_{u}^{\prime \prime}(u, \alpha) / 6=0$, denoted by $\Gamma_{\chi}$, satisfies

$$
\Gamma_{x}=\frac{\alpha\left(1-s_{f}\right)^{2}+\left(1+s_{f}\right)^{2}}{4\left(1+s_{f}\right)}>0 .
$$

As a quadratic function, one of the following two results holds for $y=g_{u}^{\prime \prime}(u, \alpha)$.

(i) $g_{u}^{\prime \prime}(u, \alpha)>0$ for all $u \in(0,1)$.

(ii) There exists a unique $\hat{u} \in(0,1)$ such that $g_{u}^{\prime \prime}(\hat{u}, \alpha)=0$, and

$$
g_{u}^{\prime \prime}(u, \alpha)<0, \text { for } u \in(0, \hat{u}) ; g_{u}^{\prime \prime}(u, \alpha)>0, \text { for } u \in(\hat{u}, 1) .
$$

For case (i), the function $y=g_{u}^{\prime}(u, \alpha)$ is strictly increasing for $u \in(0,1)$. Combining $g_{u}^{\prime}(0, \alpha)=a_{1}<0$ and $g_{u}^{\prime}(1, \alpha)<0$, we have $g_{u}^{\prime}(u, \alpha)<0$ for any $u \in(0,1)$. For case (ii), the relation (3.16) implies that $y=g_{u}^{\prime}(u, \alpha)$ decreases from $g_{u}^{\prime}(0, \alpha)<0$ to $g_{u}^{\prime}(\hat{u}, \alpha)<0$ for $u \in(0, \hat{u})$, and then increases from $g_{u}^{\prime}(\hat{u}, \alpha)<0$ to $g_{u}^{\prime}(1, \alpha)<0$. This also proves $g_{u}^{\prime}(u, \alpha)<0$, which completes the proof.

Now, we are ready to prove the uniqueness of periodic solution of system (2.8)-(2.9).

Lemma 3.3. When $\alpha \in\left(0, \alpha^{\star}\right)$, system (2.8)-(2.9) has a unique periodic solution.

Proof. The existence of the periodic solution to system (2.8)-(2.9) has been proved in Theorem 2.2 as a special case with $N=1$ and $T=2$. Now, assume that system (2.8)-(2.9) has another periodic solution. Then the existence of two periodic solutions offers two cases (See Figure 2 for illustration): For Case A, we have $h_{u}^{\prime}\left(u_{0}, \alpha\right)=1, h_{u}^{\prime}\left(u_{1}, \alpha\right) \geq 1$, which leads to $g\left(u_{0}, \alpha\right)=0$ and $g\left(u_{1}, \alpha\right) \geq 0$ with $u_{0}<u_{1}$. This contradicts the conclusion in Lemma 3.2 that $g(u, \alpha)$ is strictly decreasing with respect to $u \in(0,1)$.

For Case B, we have $h_{u}^{\prime}\left(u_{0}, \alpha\right) \geq 1, h_{u}^{\prime}\left(u_{1}, \alpha\right)=1$, which lead to $g\left(u_{0}, \alpha\right) \geq 0$ and $g\left(u_{1}, \alpha\right)=0$. Unfortunately, this does not contradict the monotonicity of function $g(u, \alpha)$. To get a contradiction, we firstly prove that $h(u, \alpha)$ is strictly increasing with respect to $\alpha \geq 0$. In fact, from (3.7), we have

$$
\bar{h}_{\alpha}^{\prime}(u, \alpha)=\frac{\left(1-s_{f}\right) u \cdot\left[Q(u, \alpha)-(1+\alpha)\left(1-s_{f}\right) u\right]}{Q^{2}(u, \alpha)}=\frac{\left(1-s_{f}\right) u(1-u)^{2}}{Q^{2}(u, \alpha)}>0 .
$$



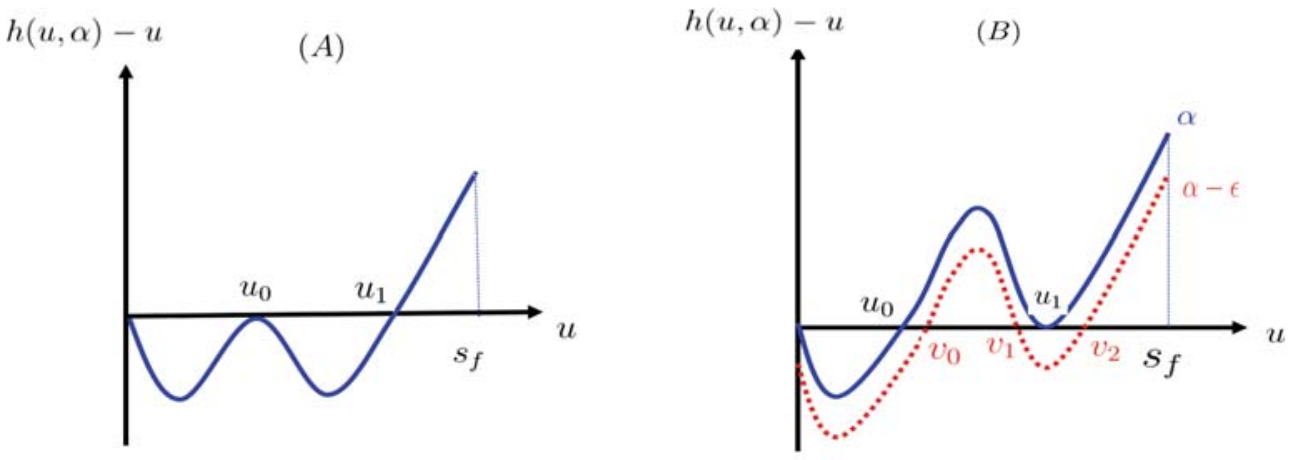

Fig. 2: Schematic for the existence of two periodic solutions of system (2.8)-(2.9).

Then, from (3.8), we get

$$
h_{\alpha}^{\prime}(u, \alpha)=\frac{d}{d \bar{h}(u, \alpha)}\left(\frac{R(\bar{h}(u, \alpha))}{S(\bar{h}(u, \alpha))}\right) \cdot \frac{\partial \bar{h}(u, \alpha)}{d \alpha}=\frac{\left(1-s_{f}\right)\left(1-\bar{h}^{2}(u, \alpha)\right)}{S^{2}(\bar{h}(u, \alpha))} \cdot \frac{\left(1-s_{f}\right) u(1-u)^{2}}{Q^{2}(u, \alpha)}>0,
$$

which proves that $h(u, \alpha)$ is strictly increasing with respect to $\alpha \geq 0$. Therefore, the function $h(u, \alpha)-u$ is also strictly increasing with respect to $\alpha \geq 0$.

Let $\epsilon>0$ be small enough such that $\alpha-\epsilon \in\left(0, \alpha^{\star}\right)$, from Lemma 3.1, we see that the curve $h(u, \alpha-$ $\epsilon)-u$ totally lies below $h(u, \alpha)-u$ except at $u=0$, which has three roots (see the right panel in Figure 2 for illustration), $u_{0}<v_{0}<v_{1}<u_{1}<v_{2}<s_{f}$ with

$$
h^{\prime}\left(v_{0}, \alpha-\epsilon\right) \geq 1, h^{\prime}\left(v_{1}, \alpha-\epsilon\right) \leq 1 \text {, and } h^{\prime}\left(v_{2}, \alpha-\epsilon\right) \geq 1,
$$

which implies that $0 \geq g\left(v_{1}, \alpha-\epsilon\right)>g\left(v_{2}, \alpha-\epsilon\right) \geq 0$, a contradiction. This completes the proof. On stability proof, (3.1) and (3.2) show that for any $\alpha>0$ and $u \in\left(s_{f}, 1\right)$, we have $h(u, \alpha)>\bar{h}(u, \alpha)>u$. Hence, if the initial value $x_{1} \in\left(s_{f}, 1\right)$, then $x_{2}=\bar{h}\left(x_{1}, \alpha\right)>x_{1}$ and $x_{3}=h\left(x_{1}, \alpha\right)>\bar{h}\left(x_{1}, \alpha\right)=x_{2}$ which yields $x_{3}>x_{2}>x_{1}$. Furthermore, we have $x_{4}=\bar{h}\left(x_{3}, \alpha\right)>x_{3}$ and $x_{5}=h\left(x_{3}, \alpha\right)>\bar{h}\left(x_{3}, \alpha\right)=x_{4}$. By induction, solution $\left\{x_{n}\right\}_{n=1}^{\infty}$ of system (2.8)-(2.9) monotonically increases to 1 for the initial value $x_{1} \in\left(s_{f}, 1\right)$. Therefore, we have the following result.

Lemma 3.4. Equilibrium $x_{1}^{\star}=1$ is locally asymptotically stable for any $\alpha>0$.

Similarly, (3.1) implies that, for $\alpha \in\left(0, \alpha_{1}^{\star}\right)$, solution $\left\{x_{n}\right\}_{n=1}^{\infty}$ of system (2.8)-(2.9) monotonically decreases to 0 if $x_{1} \in(0, \hat{x}(\alpha))$. This implies that equilibrium $x_{0}^{\star}=0$ is locally asymptotically stable for $\alpha \in\left(0, \alpha_{1}^{\star}\right)$. Furthermore, we can prove that equilibrium $x_{0}^{\star}=0$ is locally asymptotically stable for $\alpha \in\left(0, \alpha^{\star}\right)$, and the unique periodic solution initiated from $u^{\star}$ is unstable.

Proof of Theorem 2.3: From Theorem 2.2, Lemmas 3.3 and 3.4, we only need to prove the local asymptotical stability of $x_{0}^{\star}=0$ for $\alpha \in\left[\alpha_{1}^{\star}, \alpha^{\star}\right)$. Recalling that $u^{\star} \in\left(0, s_{f}\right)$ is the initial value of the unique periodic solution to system (2.8)-(2.9), we get $h(u, \alpha)<u$ for $u \in\left(0, u^{\star}\right)$, and $h(u, \alpha)>u$ for $u \in\left(u^{\star}, 1\right)$. Since

$$
\bar{h}_{u}^{\prime}(u, \alpha)=\frac{\partial}{\partial u}\left(\frac{P(u, \alpha)}{Q(u, \alpha)}\right)=\frac{\left(1-s_{f}\right)(1+\alpha)\left(1-u^{2}\right)}{Q^{2}(u, \alpha)}>0,
$$

and

$$
h_{u}^{\prime}(u, \alpha)=\frac{\partial}{\partial u}\left(\frac{R(u, \alpha)}{S(u, \alpha)}\right)=\frac{\left(1-s_{f}\right)\left(1-u^{2}\right)}{S^{2}(u, \alpha)}>0
$$

for any $u \in(0,1)$, both $h(u, \alpha)$ and $\bar{h}(u, \alpha)$ are strictly increasing in $u$.

We first show that $x_{0}^{\star}$ is stable. Solution of system (2.8)-(2.9) initiated from $x_{1} \in\left(0, u^{\star}\right)$ satisfies $x_{2}=$ $\bar{h}\left(x_{1}, \alpha\right)>x_{1}$ and $x_{3}=h\left(x_{1}, \alpha\right)<x_{1}$. Therefore, from $x_{3}<x_{1}$ and the monotonicity of $\bar{h}$, we get $x_{4}=\bar{h}\left(x_{3}, \alpha\right)<$ 
$\bar{h}\left(x_{1}, \alpha\right)=x_{2}$, and thus the monotonicity of $h$ implies that $x_{5}=h\left(x_{3}, \alpha\right)<x_{3}$, which are sufficient to prove that both $\left\{x_{2 n}\right\}_{n=1}^{\infty}$ and $\left\{x_{2 n+1}\right\}_{n=1}^{\infty}$ are strictly decreasing satisfying

$$
x_{2 n}>x_{2 n-1}>x_{2 n+1} \text {, for } n=1,2, \cdots,
$$

by induction. The relation (3.17) implies that solution $\left\{x_{n}\right\}_{n=1}^{\infty}$ achieves its maximum at $x_{2}=\bar{h}\left(x_{1}, \alpha\right)$. Hence, for any given $\epsilon \in\left(0, u^{\star}\right)$, take $\delta=\min \left\{\epsilon, \bar{h}^{-1}(\epsilon, \alpha)\right\}$, then for any $x_{1}<\delta$, we have

$$
x_{n}\left(1, x_{1}\right) \leq \bar{h}\left(x_{1}, \alpha\right)<\bar{h}(\delta, \alpha)<\epsilon,
$$

i.e., equilibrium $x_{0}^{\star}$ is stable.

Regarding the attractivity of $x_{0}^{\star}$, the relation (3.17) implies that to prove every solution with $x_{1} \in(0, \delta) \subset$ $\left(0, u^{\star}\right)$ of system (2.8)-(2.9) goes to zero, we only need to prove

$$
\lim _{n \rightarrow \infty} x_{2 n}\left(1, x_{1}\right)=0 .
$$

Otherwise, the $\lim _{n \rightarrow \infty} x_{2 n}\left(1, x_{1}\right)=l \neq 0$ offers a periodic solution $x_{n}\left(1, \bar{h}^{-1}(l, \alpha)\right)$, a contradiction to the uniqueness of the periodic solution to system (2.8)-(2.9). Finally, the instability of the unique periodic solution initiated from $u^{*}$ follows from the fact that $h(u, \alpha)>u$ for $u \in\left(u^{*}, 1\right)$, and $h(u, \alpha)<u$ for $u \in\left(0, u^{*}\right)$, which implies that no matter how close the initial value $x_{1}$ to $u^{*}$ is, solutions initiated from $x_{1}$ will gradually drift away from $u^{*}$.

\subsection{Proof of Theorem 2.4}

We claim that when $\alpha \geq \alpha^{\star}, h(u, \alpha)>u$ for $u \in(0,1)$. Since $h(u, \alpha)>u$ for $u \in\left[s_{f}, 1\right)$, it suffices to prove

$$
h(u, \alpha)>u \text { for } u \in\left(0, s_{f}\right) .
$$

When $\alpha \geq \alpha^{\star}$, we have $h_{u}^{\prime}(0, \alpha) \geq 1$. If (3.18) does not hold, then there exists $u_{0} \in\left(0, s_{f}\right)$, such that either

$$
h\left(u_{0}, \alpha\right)=u_{0}, h(u, \alpha)>u \text { for all } u \in\left(0, u_{0}\right) \cup\left(u_{0}, s_{f}\right),
$$

or $h\left(u_{0}, \alpha\right)<u_{0}$ and hence there exists $u_{1}$ and $u_{2}$ satisfying $u_{1}<u_{2}<s_{f}$, such that

$$
\begin{gathered}
h\left(u_{1}, \alpha\right)=u_{1}, h\left(u_{2}, \alpha\right)=u_{2}, \\
h(u, \alpha)>u \text { for } u \in\left(0, u_{1}\right) \cup\left(u_{2}, s_{f}\right) \text { and } h(u, \alpha)<u \text { for } u \in\left(u_{1}, u_{2}\right) .
\end{gathered}
$$

For both cases, as what have been proved in Lemma 3.3 that $h(u, \alpha)$ is strictly increasing with respect to $\alpha \geq 0$, we can choose $\epsilon>0$ small enough such that $h(u, \alpha-\epsilon)-u$ has two roots, denoted by $v_{1}$ and $v_{2}$ satisfying $v_{1}<v_{2}$, and $h^{\prime}\left(v_{1}, \alpha-\epsilon\right) \leq 1$ and $h^{\prime}\left(v_{2}, \alpha-\epsilon\right) \geq 1$. Or, equivalently, $g\left(v_{1}, \alpha-\epsilon\right) \leq 0$ and $g\left(v_{2}, \alpha-\epsilon\right) \geq 0$, which contradicts to the fact that the function $g(u, \alpha)$ is strictly decreasing shown in Lemma 3.2. Similar figure as Figure 2 can be plotted for illustration and we omit here.

The local asymptotical stability of $x_{1}^{\star}=1$ has been proved in Lemma 3.4. To prove that $x_{1}^{\star}=1$ is also a global attractor, we only need to prove that $x_{0}^{\star}=0$ is unstable since the conclusion $h(u, \alpha)>u$ for all $u \in(0,1)$ excludes the existence of periodic solution to system (2.8)-(2.9). In fact, when $\alpha \geq \alpha^{\star}>\alpha_{1}^{\star}$, for any $u \in\left(0, s_{f}\right)$, we have $\bar{h}(u, \alpha)>h(u, \alpha)>u$, which implies that $\bar{h}\left(x_{1}, \alpha\right)>h\left(x_{1}, \alpha\right)>x_{1}$, i.e., $x_{2}>x_{3}>x_{1}$ with $x_{1} \in\left(0, s_{f}\right)$. Therefore, $x_{4}=\bar{h}\left(x_{3}, \alpha\right)>\bar{h}\left(x_{1}, \alpha\right)=x_{2}$ and $x_{5}=h\left(x_{3}, \alpha\right)>x_{3}$ which are sufficient to claim that both $\left\{x_{2 n}\right\}_{n=1}^{\infty}$ and $\left\{x_{2 n+1}\right\}_{n=1}^{\infty}$ are strictly increasing satisfying $x_{2 n}>x_{2 n+1}>x_{2 n-1}$, for $n=1,2, \cdots$ by induction. Hence, any solution initiated from $\left(0, s_{f}\right)$ run away from $x_{0}$ and $x_{0}^{\star}=0$ is unstable. This completes the proof. 


\section{Concluding remarks}

Theorems 2.3 and 2.4 show the existence of the release ratio threshold $\alpha^{\star}$ : below which system (2.8)-(2.9) admits a unique unstable periodic solution, and above which Wolbachia invasion is guaranteed to be successful as long as infected mosquitoes are seeded initially. To verify this result numerically, we assume that Wolbachia infection causes a mild fitness cost to the host mosquito with $s_{f}=0.3$, and hence $\alpha^{\star} \approx 1.041$. Numerical calculations using Matlab yield $u^{\star} \approx 0.132$ when the release ratio $\alpha=0.5<\alpha^{\star}$, and hence $\bar{h}\left(u^{\star}\right) \approx 0.155$ by equation (3.7), from which we artificially plot the unstable periodic solution in Figure 3(A) to show the local asymptotical stability of $x_{0}^{\star}=0$ and $x_{1}^{\star}=1$. Several orbits of system (2.8)-(2.9) initiated above or below $u^{\star}$ are also plotted in Figure 3(A). Increasing of $\alpha$ to $1.05>\alpha^{\star}$ drives the periodic solution to disappear and the Wolbachia invasion equilibrium $x_{1}^{\star}=1$ is globally asymptotically stable, which is numerically verified in Figure 3(B).
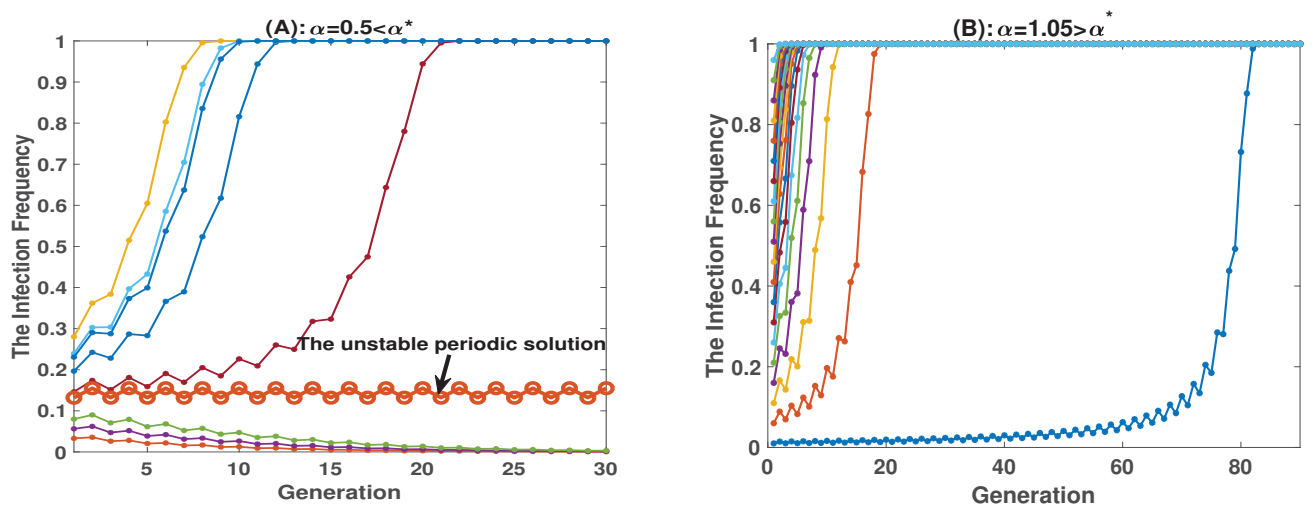

Fig. 3: Numerical verification of results in Theorems 2.3 and 2.4 with $s_{f}=0.3$.
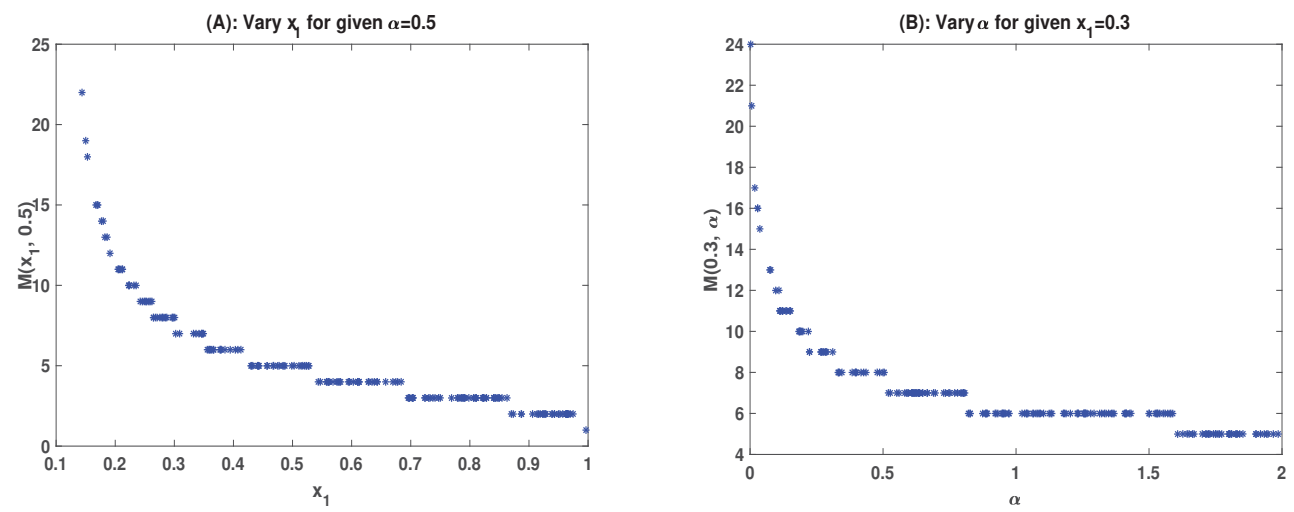

Fig. 4: Extra releases of Wolbachia males speed up the Wolbachia invasion.

We end the whole paper with numerical demonstrations on how extra releases of Wolbachia male mosquitoes speed up the Wolbachia invasion. To this end, for given $s_{f}=0.3$, we explore the quantitative behavior of the first generation point, denoted by $M=M\left(x_{0}, \alpha\right)$, satisfying

$$
x_{n}<0.98 \text { for } n=1,2, \cdots, M-1 \text {, but } x_{M} \geq 0.98 \text {, }
$$

where we claim the success of Wolbachia invasion once the infection frequency is greater than or equals to 0.98. The smaller the value $M$, the faster the Wolbachia invasion. Figure 3(A) shows that when $x_{1}<u^{\star}$ and $\alpha<$ 
$\alpha^{\star}, M\left(x_{1}, \alpha\right)=\infty$, i.e., Wolbachia invasion is impossible. To see how $M\left(x_{1}, \alpha\right)$ varies with the initial frequency $x_{1}$, we plot $M\left(x_{1}, 0.5\right)$ against $x_{1}$ in Figure 4(A) by solving system (2.8)-(2.9) under 200 initial values which are randomly selected in $(0.14,1) \subset\left(u^{\star}, 1\right)$. The increase of $x_{1}$ causes a step-like increase of the Wolbachia invasion speed: a sharp decrease of $M\left(x_{1}, 0.5\right)$ from 25 to 10 when $x_{1}$ goes from 0.14 to 0.23 , and a mild decrease of $M\left(x_{1}, 0.5\right)$ from 9 to 2 when $x_{1}$ goes from 0.24 to 0.97 .

Similarly, for given $x_{1}=0.3$, the increase of $\alpha$ drives a step-like decrease of $M(0.3, \alpha)$ which decreases sharply from 24 to 10 when the release ratio goes from 0.003 to 0.22 . However, further increase of $\alpha$ from 0.23 to 2 does not significantly speed up the Wolbachia invasion since $M(0.3,0.23)=10$ and $M(0.3,2)=5$. This phenomenon implies the existence of an optimal release ratio for given $s_{f}$ and $x_{1}$, for example, the above numerical trials imply that the optimal release ratio is about 0.22 when $x_{1}=0.3$ and $s_{f}=0.3$. To maximize the economic benefits when designing release strategies, the release ratio is better to be kept close to the optimal release ratio.

Acknowledgement: This work is supported by National Natural Science Foundation of China (11971127, 12071095, 11631005), Program for Changjiang Scholars and Innovative Research Team in University (IRT_16R16).

Conflict of interest: Authors state no conflict of interest.

\section{References}

[1] H.N. Aida, H. Dieng, A.T. Nurita, et al. The biology and demographic parameters of Aedes albopictus in northern peninsular Malaysia, Asian Pac. J. Trop. Biomed. 1(2011), 472-477.

[2] G. Bian, Y. Xu, P. Lu, et al. The endosymbiotic bacterium Wolbachia induces resistance to dengue virus in Aedes aegypti, PLoS Pathog. 6(2010), no. 4, e1000833.

[3] G. Bian, D. Joshi, Y. Dong, et al. Wolbachia invades Anopheles stephensi populations and induces refractoriness to plasmodium infection, Science, 340(2013), 748-751.

[4] J.S. Brownstein, E. Hett, S.L. O'Neill, The potential of virulent Wolbachia to modulate disease transmission by insects, J. Invertebr. Pathol. 84(2003), no. 1, 24-29.

[5] L. Cai, S. Ai and J. Li, Dynamics of mosquitoes populations with different strategies for releasing sterile mosquitoes, SIAM J. Appl. Math. 74(2014), no. 6, 1786-1809.

[6] E. Caspari and G.S. Watson, On the evolutionary importance of cytoplasmic sterility in mosquitoes, Evolution, 13(1959), 568-570.

[7] L.M. Field, A.A. James, M. Turelli, et al. Microbe-induced cytoplasmic incompatibility as a mechanism for introducing transgenes into arthropod populations, Insect Mol. Biol. 8(1999), no. 2, 243-255.

[8] P.E.M. Fine, On the dynamics of symbiote-dependent cytoplasmic incompatibility in Culicine mosquitoes. J. Invertebr. Pathol. 31(1978), 10-18.

[9] M.G. Grech, Ludueha-Almeida F, Almiron W R. Bionomics of Aedes aegypti subpopulation (Diptera: Culicidae) from Argentina, J. Vector Ecol. 35(2011), no. 2, 277-285.

[10] S. He, X. Zhang, J. Liang, et al. Multiscale modelling the effects of $\mathrm{Cl}$ genetic evolution in mosquito population on the control of dengue fever, Sci. Rep. 7(2017), no. 1, 13895.

[11] A.A. Hoffmann, B.L. Montgomery, J. Popovici, et al. Successful establishment of Wolbachia in Aedes populations to suppress dengue transmission, Nature, 476(2011), no. 7361, 454-457.

[12] L. Hu, M. Huang, M. Tang, et al. Wolbachia spread dynamics in stochastic environments, Theor. Popul. Biol. 106(2015), 32-44.

[13] L. Hu, M. Tang, Z. Wu, et al. The threshold infection level for Wolbachia invasion in random environments, J. Differ. Equations, 266(2019), no. 7, 4377-4393.

[14] M. Huang, L. Hu and J. Yu, Wolbachia infection dynamics by reaction-diffusion equations, Sci. China Math. 58(2015), no. 1, 77-96.

[15] M. Huang, J. Luo, L. Hu, et al. Assessing the efficiency of Wolbachia driven Aedes mosquito suppression by delay differential equations, $J$ Theor. Biol. 440(2018), no. 7, 1-11.

[16] M. Huang, M. Tang, J. Yu, et al. A stage structured model of delay differential equations for Aedes mosquito population suppression, Discrete Contin. Dyn. Syst. Ser. A, 40(2020), no. 6, 3467-3484.

[17] M. Huang, L. Hu, B. Zheng, Comparing the efficiency of Wolbachia driven Aedes mosquito suppression strategies, J. Appl. Anal. Comput. 9(2019), no. 1, 211-230. 
[18] Y. Hui, G. Lin, Y. Yu, et al. A delayed differential equation model for mosquito population suppression with sterile mosquitoes, Discrete Contin. Dyn. Syst. Ser. B, 25(2020), no. 12, 4659-4676.

[19] Y. Li, L.A. Baton, D. Zhang et al. Reply to: Issues with combining incompatible and sterile insect techniques, Nature, 590(2021), E3-E5.

[20] J. Li, L. Cai and Y. Li, Stage-structured wild and sterile mosquito population models and their dynamics, J. Biol. Dyn. 11(2016), 79-101.

[21] Y. Li and X. Liu, An impulsive model for Wolbachia infection control of mosquito-borne diseases with general birth and death rate functions, Nonlinear Anal. Real World Appl. 37(2017), 412-432.

[22] Y. Li and X. Liu, A sex-structured model with birth pulse and release strategy for the spread of Wolbachia in mosquito population, J. Theor. Biol. 448(2018), 53-65.

[23] Y. Li and X. Liu, Modeling and control of mosquito-borne diseases with Wolbachia and insecticides, Theor. Popul. Biol. 132(2020), 82-91.

[24] C.J. Mcmeniman, R.V. Lane, B.N. Cass, et al. Stable introduction of a life-shortening Wolbachia infection into the mosquito Aedes aegypi, Science, 323(2009), 141-144.

[25] Y. Shi and J. Yu, Wolbachia infection enhancing and decaying domains in mosquito population based on discrete models, J. Biol. Dyn. 14(2020), no. 1, 679-695.

[26] M. Turelli and A.A. Hoffmann, Rapid spread of an inherited incompatibility factor in California Drosophila, Nature, 353(1991), no. 6343, 440-442.

[27] M. Turelli, Evolution of incompatibility-inducing microbes and their hosts, Evolution, 48(1994), no. 5, $1500-1513$.

[28] M. Turelli, Cytoplasmic incompatibility in populations with overlapping generations, Evolution, 64(2010), no. 1, $232-241$.

[29] T. Walker, P.H. Johnson, L.A. Moreira, et al. The wMel Wolbachia strain blocks dengue and invades caged Aedes aegypti populations, Nature, 476(2011), no. 7361, 450-453.

[30] Z. Xi and S.L. Dobson, Characterization of Wolbachia transfection efficiency by using microinjection of embryonic cytoplasm and embryo homogenate, Appl. Environ. Microbiol. 71(2005), no. 6, 3199-3204.

[31] Z. Xi, J.L. Dean, C.C. Khoo, et al. Generation of a novel Wolbachia infection in Aedes albopictus (Asian tiger mosquito) via embryonic microinjection, Insect Biochem. Mol. Biol.35(2005), no. 8, 903-910.

[32] Z. Xi, C.C. Khoo and S.L. Dobson, Wolbachia establishment and invasion in an Aedes aegypti laboratory population, Science, 310(2005), 326-328.

[33] Z. Xi, C.C. Khoo and S.L. Dobson, Interspecific transfer of Wolbachia into the mosquito disease vector Aedes albopictus, Proc. Royal Soc. B, 273(2006), no. 1592, 1317-1322.

[34] Y. Yu, Modelling mosquito population suppression based on delay differential equations, SIAM J. Appl. Math. 78(2018), no. 6, 3168-3187.

[35] J. Yu and B. Zheng, Modeling Wolbachia infection in mosquito population via discrete dynamical models, J. Differ. Equ. Appl. 25(2019), no. 11, 1549-1567.

[36] J. Yu and J. Li, Dynamics of interactive wild and sterile mosquitoes with time delay, J. Biol. Dyn. 13(2019), no. 1, 606-620.

[37] J. Yu, Existence and stability of a unique and exact two periodic orbits for an interactive wild and sterile mosquito model, J. Differ. Equations, 269(2020), no. 12, 10395-10415.

[38] J. Yu and J. Li, Global asymptotic stability in an interactive wild and sterile mosquito model, J. Differ. Equations, 269(2020), no. 7, 6193-6215.

[39] X. Zhang, Q. Liu and H. Zhu, Modeling and dynamics of Wolbachia-infected male releases and mating competition on mosquito control, J. Math. Biol. 81(2020), 1-34.

[40] B. Zheng, M. Tang and J. Yu, Modeling Wolbachia spread in mosquitoes through delay differential equations, SIAM J. Appl. Math. 74(2014), no. 3, 743-770.

[41] B. Zheng, M. Tang, J. Yu, et al. Wolbachia spreading dynamics in mosquitoes with imperfect maternal transmission, J. Math. Biol. 76(2018), no. 1-2, 235-263.

[42] B. Zheng, J. Yu, Z. Xi, et al. The annual abundance of dengue and Zika vector Aedes albopictus and its stubbornness to suppression, Ecol. Model. 387(2018), no. 10, 38-48.

[43] B. Zheng, W. Guo, L. Hu, et al. Complex Wolbachia infection dynamics in mosquitoes with imperfect maternal transmission, Math. Biosci. Eng. 15(2018), no. 2, 523-541.

[44] B. Zheng, J. Yu and J. Li, Modeling and analysis of the implementation of the Wolbachia incompatible and sterile insect technique for mosquito population suppression, SIAM J. Appl. Math. 81(2021), no. 2, 718-740.

[45] B. Zheng, L. Chen and Q. Sun, Analyzing the control of dengue by releasing Wolbachia-infected male mosquitoes through a delay differential equation model, Math. Biosci. Eng. 16(2019), no. 5, 5531-5550.

[46] B. Zheng, X. Liu, M. Tang, et al, Use of age-stage structural models to seek optimal Wolbachia-infected male mosquito releases for mosquito-borne disease control, J. Theor. Biol. 472(2019), 95-109.

[47] X. Zheng, D. Zhang, Y. Li, et al, Incompatible and sterile insect techniques combined eliminate mosquitoes, Nature, 572(2019), 56-61. 